2

\title{
3 How plants cope with UV-B: from perception to response
}

4

5 Ruohe Yin $^{1}$, and Roman Ulm ${ }^{1,2}$

6

$7{ }^{1}$ Department of Botany and Plant Biology, Section of Biology, Faculty of Sciences, 8 University of Geneva, CH-1211 Geneva 4, Switzerland

$9{ }^{2}$ Institute of Genetics and Genomics of Geneva (iGE3), University of Geneva, Geneva, 10 Switzerland

Corresponding author: Ulm, Roman (roman.ulm@unige.ch)

14 Short title: UV-B signaling in plants 
15 Abstract

16 Ultraviolet-B radiation (UV-B) is an intrinsic part of the solar radiation that reaches the earth's surface and affects the biosphere. Plants have evolved a specific UV-B signaling pathway mediated by the UVR8 photoreceptor that regulates growth, development, and acclimation. Major recent advances have contributed to our understanding of the UVR8 photocycle, UV-B-responsive protein-protein interactions, regulation of UVR8 subcellular localization, and UVR8-regulated physiological responses. Here, we review the latest progress in our understanding of UVR8 signaling and UV-B responses, which includes studies in the unicellular alga Chlamydomonas reinhardtii and the flowering plant Arabidopsis. 


\section{Introduction}

Plants adjust their growth and development according to ambient light conditions that can dynamically change. Plants have evolved a set of light-activated molecules, known as photoreceptors, which monitor light quality, quantity, direction, and duration. Phytochromes (phyA-phyE in Arabidopsis) are red/far-red light photoreceptors, whereas cryptochromes (cry1 and cry2), phototropins (phot1 and phot2), and Zeitlupe family members (ZTL, FKF1, and LKP2) are UV-A/blue light photoreceptors, and UVR8 is a UV-B photoreceptor $[1,2]$. Light influences a vast number of plant physiological and developmental programs, ranging from seed germination to the transition from vegetative growth to flowering.

UV-B (280-315 nm) induces plant photomorphogenesis as well as UV-B acclimation responses, which enable plants to tolerate UV-B stress [3,4]. A comprehensive molecular picture of the initial events in the UVR8 signaling pathway has begun to emerge. In the current review, we will describe recent key findings concerning the molecular events in UVR8-mediated signaling and responses, and attempt to place these findings into a broader context. Other reviews on this topic have been published recently, and present complementary perspectives on the mechanisms and functional implications of UV-B perception and signaling [4-7].

\section{UVR8 photoreceptor-mediated UV-B responses}

The most commonly described UVR8-mediated UV-B responses in Arabidopsis are inhibition of hypocotyl growth, accumulation of flavonols and anthocyanins, and changes in gene expression or protein accumulation [8-12] (Figure 1). Moreover, UVR8 signaling significantly contributes to UV-B acclimation responses and the establishment of UV-B tolerance $[3,8,10]$. UV-B acclimation can be associated with UVR8-activated gene expression associated with flavonoid biosynthesis, DNA repair, and protection against oxidative stress and photoinhibition [9,10,13-15]. Various additional physiological responses have been linked to UVR8 photoreceptor activity, including entrainment of the circadian clock [16], phototropism [17], downward leaf curling [18], and stomatal opening [19]. Furthermore, UVR8-mediated UV-B signaling influences defense responses [20], leaf development [21], salt stress tolerance [22], thermomorphogenesis [23“"], shade avoidance responses [24,25], and auxin signaling [17,24]. It is of note, however, that $u v r 8$ and wild-type seedlings are indistinguishable in the absence of UV-B [8-10].

Although the majority of the current knowledge has been generated using Arabidopsis as a model system, UVR8-induced responses and UV-B acclimation was also demonstrated in 
the green alga Chlamydomonas reinhardtii (herein referred to as Chlamydomonas) $\left[26^{*}, 27^{\circ}\right]$. In Chlamydomonas, UV-B acclimation was associated with protection of the photosynthetic apparatus through induction of non-photochemical quenching, which allows dissipation of harmful excess light energy as heat (qE) and prevents photodamage [28]. Indeed, UVR8 signaling enhances the capacity of $\mathrm{qE}$ in Chlamydomonas, representing an anterograde link between UV-B photoreceptor-mediated signaling in the nucleo-cytosolic compartment and photoprotective regulation of photosynthetic activity in the chloroplast [26“]. Interestingly, the molecular effectors that are induced by UVR8 signaling and underlie UVR8-mediated photoprotection are partially different from those induced by high light that are regulated by the blue light photoreceptor phototropin $\left[26^{*}, 28\right]$.

For a number of reported UV-B responses, it is still unknown whether the response studied is UVR8 photoreceptor-dependent or not. Given the availability of Arabidopsis $u v r 8$ lines [8-10], a Chlamydomonas $u v r 8$ line [26“" ], and the possibility to create UVR8 null mutants in other plant species [29], it should be straightforward to determine the relationship between UV-B response phenotypes and UVR8 signaling, which serves to both verify existing and discover additional UVR8-regulated responses.

\section{The core of the UVR8 photocycle: a short summary}

In contrast to those photoreceptors that perceive visible light, UVR8 UV-B photon reception is accomplished by specific tryptophans intrinsic to the UVR8 protein in lieu of a bound chromophore [1,30-32]. In its ground state, UVR8 forms homodimers which are held together by electrostatic interactions between charged amino acids at the dimer interface [1,10,30,31]. In response to UV-B, these interactions are broken and UVR8 homodimers dissociate into active monomers [1,30-39]. However, rather than embodying a simple UV-B switch, UVR8 establishes a homodimer/monomer photoequilibrium under natural conditions [40]. UVR8 monomers bind to CONSTITUTIVELY PHOTOMORPHOGENIC 1 (COP1) to initiate UVB signaling $\left[10,12,41^{\circ}, 42-44\right]$. Together with its accessory SUPPRESSOR OF PHYA-105 (SPA) proteins, COP1 functions as a CULLIN4-DAMAGED DNA BINDING PROTEIN 1 (CUL4-DDB1)-based E3 ubiquitin ligase, which is a well-known key repressor of photomorphogenesis [45]. In apparent contrast to this role, COP1 is required for UV-Binduced responses [46]. Downstream of UVR8 and COP1, the bZIP transcription factors ELONGATED HYPOCOTYL 5 (HY5) and HY5 HOMOLOG (HYH) are required for the transcriptional regulation of a subset UV-B-regulated genes [9,13,47-49]. Genes encoding the WD40-repeat proteins REPRESSOR OF UV-B PHOTOMORPHOGENESIS 1 (RUP1) and 
RUP2 are induced by UV-B in a UVR8-, COP1-, and HY5-dependent manner [50]. RUP1 and RUP2 interact directly with UVR8 and facilitate the reversion of UVR8 monomers to homodimers to balance the signaling pathway [50,51].

\section{Two distinct domains of UVR8 mediate UV-B-dependent interaction with COP1}

UVR8 is comprised of two distinct domains: a seven-bladed $\beta$-propeller core domain, formed by RCC1-repeats, and a C-terminal area of 27 amino acids, coined the $\mathrm{C} 27$ domain [1,30,31,43]. Active UVR8 interacts via both these domains with the C-terminal WD40-repeat domain of COP1 $\left[1,10,41^{\circ}, 43\right]$, which also forms a seven-bladed $\beta$-propeller structure [52]. A truncated UVR8 core lacking the C27 domain is able to interact with COP1 in a UV-Bdependent manner, although this interaction is weaker than that of wild-type UVR8 [41 ${ }^{\circ}$. Thus, the UVR8 C27 domain, which contains what is referred to as a VP (valine-proline) domain, contributes to the interaction between UVR8 and COP1 $\left[41^{\circ}, 43,53\right]$. A VP domain possesses the core sequence V-P-E/D- $\varphi-\mathrm{G}$ (where $\varphi=$ a hydrophobic residue) and can be identified in several COP1-interacting proteins, where it mediates interaction with the COP1 WD40-repeat domain $\left[41^{\circ}, 54\right]$. Furthermore, a structural basis for this interaction has recently been described [52]. Interestingly, a truncated version of UVR8 containing only the C27 domain is able to interact constitutively with COP1 in both transgenic plants and yeast, whereas the non-UV-B-activated wild-type UVR8 homodimer does not interact with COP1, despite the presence of the $\mathrm{C} 27$ domain $\left[41^{\circ}, 43\right]$. Thus, upon UV-B-induced monomerisation, the $\mathrm{C} 27$ domain appears to be released from structural constraints imposed by the UVR8 core. Furthermore, it can be envisioned that the two COP1 interaction surfaces, namely the $\beta$ propeller core and the C27 VP domain, which are not accessible in the UVR8 homodimer, are exposed in UV-B-activated UVR8 monomers. The $\beta$-propeller domain allows UV-Bdependent interaction of UVR8 with COP1 and this interaction is further stabilized by the C27 domain that affects COP1 activity [ $\left.41^{\circ}\right]$. The effects on COP1 activity may be the result of competitive binding of the C27 VP domain of UVR8 with similar VP domains present in COP1 substrate proteins, such as HY5 [52,54]. In darkness and low-light in particular, HY5 is ubiquitinated by COP1 and consequently targeted for proteasome-mediated degradation [45]. However, under UV-B, HY5 is stabilized [10,55]. Thus, the C27 VP domain of UVR8 may compete with the VP domain of HY5 for COP1 binding, resulting in the protection of HY5 from degradation. This HY5 stabilization mechanism is further supported by the UV-Bresponsive formation of UVR8-COP1-SPA complexes, which disrupt and therefore inactivate the active CUL4-DDB1 ${ }^{\text {COP1-SPA }}$ E3 ubiquitin ligase [44,55]. 
It is interesting to note that the negative regulators RUP1 and RUP2 are WD40-repeat proteins expected to form seven-bladed $\beta$-propeller structures comparable to the COP1 Cterminal domain [50]. Similar to the COP1 WD40 repeats, RUP1 and RUP2 interact with the C27 VP domain of UVR8 $\left[41^{\circ}, 43,50\right]$. However, in contrast to COP1, RUP1 and RUP2 have been found to also interact with the UVR8 homodimeric ground state in vivo $\left[41^{\circ}, 50\right]$. This interaction agrees with the role of RUP1 and RUP2 in facilitating the reversion of UVR8 monomers to homodimers [51]. The fact that COP1, RUP1, and RUP2 interact with the UVR8 C-terminus under UV-B implies that RUP1 and RUP2 compete with COP1 for binding to the UVR8 C27 VP domain, which would provide a further regulatory level in VP domainmediated interactions with COP1. However, it is important to note that in Arabidopsis, UVR8 redimerization is comparable in both copl and wild-type, as well as in both rupl rup2 copl and rup1 rup2 [51'], which does not support a mechanism in which RUP1- and RUP2mediated disruption of the UVR8-COP1 interaction per se allows UVR8 redimerization. Conversely, RUP1 and RUP2 are able to facilitate UVR8 redimerization through direct interaction, which is independent of COP1. It remains to be determined whether the release of COP1 is caused by UVR8 redimerization alone or the result of competition between RUP1/RUP2 and COP1 for the C27 VP domain binding site of UVR8.

\section{UVR8 nuclear accumulation in response to UV-B}

UVR8 is localized primarily in the cytosol and to a lesser extent in the nucleus of Arabidopsis seedlings. In response to UV-B, activated UVR8 monomers accumulate in the nucleus $\left[56,57^{\circ}, 58^{\circ}\right]$. Recent findings further support the notion that UVR8 nuclear localization is required for UV-B signaling, which agrees with past studies $[9,10,56]$. The fusion of glucocorticoid receptor (GR) to UVR8 retains the GR-UVR8 protein in the cytosol in the absence of dexamethasone treatment, and thus prevents UV-B signaling and the activation of UV-B responses $\left[57^{\circ}, 58^{\circ}\right]$. Although cytosolic GR-UVR8 can be UV-B-activated, meaning the GR-UVR8 homodimer monomerizes and interacts with COP1, UV-B-responsive photomorphogenesis is only apparent when GR-UVR8 is able to enter the nucleus in the presence of dexamethasone $\left[57^{\circ}, 58^{\circ}\right]$. It should be noted that a large portion of total UVR8 is present in the cytosol even under UV-B $\left[56,57^{\circ}, 58^{\circ}\right]$, which suggests that cytosolic UVR8 may have a currently undescribed function.

No obvious nuclear localization signal (NLS) is apparent in UVR8, which raises the question of how UVR8 accumulates in the nucleus. Interestingly, UV-B-triggered UVR8 nuclear accumulation was found to be largely abolished in $\operatorname{cop} 1\left[57^{\circ}\right]$. Thus, COP1 may be 
responsible for UVR8 nuclear import, as the protein has both an intrinsic NLS and nuclear export signal (NES) sequence, displays light-regulated nucleo-cytosolic partitioning as well as specific UV-B-mediated nuclear accumulation, and interacts with UVR8 in a UV-Bdependent manner $[10,45,46,59]$. As an alternative to such a "piggy-back" co-import mechanism, another possibility is that UVR8 enters the nucleus in a COP1-independent manner, which may proceed either by diffusion of UVR8 monomers or by interaction of UVR8 with a presently unknown NLS-containing protein, with nuclear COP1 required to retain activated UVR8 in the nucleus (Figure 2). It is of note that in transgenic lines expressing nuclear-localized NLS-UVR8 or dexamethasone-treated GR-UVR8 fusion proteins in a copl background, UV-B treatment cannot trigger UV-B responses in the absence of a functional COP1 $\left[5^{\circ}\right]$. Thus, regardless of the exact mechanism behind UVR8 nuclear accumulation, it is clear that COP1 plays a dual role in mediating UVR8 nuclear accumulation and UVR8 signaling.

\section{Regulation of gene expression by UVR8}

The expression of several genes is rapidly regulated by the UVR8 pathway in response to UVB $[9,10]$. Indeed, at present, all UVR8-mediated UV-B responses can be linked to a transcriptional response. However, the molecular mechanism by which UVR8 activation leads to transcriptional changes via the UVR8 signal transduction pathway is not well characterized. UV-B-induced genes were found to be often associated with increased histone $\mathrm{H} 3$ acetylation at lysines $\mathrm{K} 9$ and/or K14, and pharmacological inhibition of histone acetylation impaired transcriptional induction of a number of tested UV-B target genes; however, no specific histone acetyltransferase involved in this process could be identified [60]. It has been reported that UVR8 is a histone-binding protein that binds constitutively to chromatin of a number of UVR8-regulated genes [9,61]. However, the functional significance of UVR8 chromatin association remains to be established. Moreover, chromatin binding of UVR8 has recently been questioned [62], suggesting that the effect of UVR8-COP1 on gene expression is through the regulation of transcription factors and does not involve direct association of UVR8 with chromatin at target genes.

HY5 and HYH form homodimers and heterodimers and enact the majority of UVR8regulated genes under UV-B [9,13,47,49,63,64]. Expression of $H Y 5$ and $H Y H$ is induced by UV-B and both HY5 and HYH are stabilized post-translationally under UV-B $[9,10,49,55]$. HY5 binds to a T/G-box cis-element in its own promoter and promotes HY5 expression in response to UV-B [48]. Moreover, HY5 and HYH also bind to the promoters of other tested 
UV-B-regulated genes, including $M Y B 12, C H S, R U P 1, R U P 2, C O P 1$, and $B B X 24$ [48]. HY5 binding to chromatin at target promoters is enhanced by UV-B and dependent on UVR8 [48]. In addition to HY5 and HYH, several other transcription factors were linked to UV-Binduced gene expression, and their mode-of-action is potentially downstream of the UVR8COP1 signaling pathway. The transcription factor FHY3 binds to a regulatory element in the COP1 promoter that is distinct from that of HY5 and contributes to UV-B-induced expression of COP1 [63]. Indeed, HY5 and FHY3 interact and UV-B negatively regulates this interaction [63]. The B-box protein BBX24, a further UV-B-induced transcription factor, also interacts with HY5; however, BBX24 was found to function as a negative regulator of UV-B responses [65]. The mechanistic details of this regulatory network involving HY5 and its interacting transcription factors in the context of the UVR8-mediated UV-B signal transduction pathway remain to be determined.

Recent work is revealing the impact of UV-B signaling on transcriptional responses to other environmental signals, namely shade (reduced red/far-red ratio) and temperature $\left[233^{*}, 24\right]$. It was suggested that the bHLH transcription factors PHYTOCHROME INTERACTING FACTOR 4 (PIF4) and PIF5 were affected by UVR8 signaling, which contributes to the inhibition of shade avoidance responses by UV-B [24]. Indeed, UV-B triggers the degradation of PIF4 and PIF5, which may block up-regulation of auxin biosynthesis that is required for stem elongation in the shade avoidance response [24]. In addition, UV-B leads to stabilization of the gibberellic acid-regulated growth-repressing DELLA proteins, which may inhibit the remaining PIF activity under UV-B [24].

Recently, UVR8 has also been found to inhibit high temperature-induced auxin signaling and stem elongation (thermomorphogenesis) in response to UV-B [23"*]. Alongside decreased PIF4 transcript accumulation that reduces PIF4 abundance, UV-B also stabilizes the bHLH factor LONG HYPOCOTYL IN FAR RED (HFR1), which can bind to and inhibit PIF4 function by forming non-DNA binding heterodimers $[23 * 66]$. In contrast to the involvement of UV-B signaling in the shade avoidance response, UV-B inhibition of thermomorphogenesis does not seem to involve PIF4 degradation or DELLA stabilization $[23 * 24]$.

\section{Evolutionary conservation of the UVR8-COP1 UV-B signaling pathway}

Identifying features of UVR8 are the three key tryptophans at the dimer interface (Trps-233, 285, and -337 in Arabidopsis) that exist in conserved GWRHT/S domains (amino acid single letter code; note that the charged arginine residues next to the tryptophans are crucial to 
maintain UVR8 homodimers), and the C27 VP domain [4,67]. UVR8 appeared in the green lineage and displays a high degree of conservation in photosynthetic organisms, from green algae to higher plants $[1,4,67]$. Aside from the seagrass Zostera marina, which is a marine angiosperm where UVR8 was apparently lost as the organism adapted to growth in the sea [68], UVR8 is broadly present in green algae, bryophytes, lycophytes, and angiosperms [67]. UVR8 is not present, however, in prasinophytes, red algae, and glaucophytes [67]. Indeed, the Chlamydomonas ortholog of UVR8 was found to monomerize in response to UV-B, redimerize to revert to the homodimeric ground state, and interact with the Chlamydomonas ortholog of COP1 in a UV-B-dependent manner $\left[26^{\circ}, 27^{\circ}\right]$, indicating that the core UVR8 photocycle is well-conserved between green algae and flowering plants.

\section{Conclusions}

Since the identification of UVR8 as the UV-B photoreceptor, significant progress has been made in understanding the mechanistic action of UVR8. The UV-B-dependent interaction between UVR8 and COP1 has emerged as a key mechanism in UV-B signaling, and new roles for this interaction in the nuclear accumulation of UVR8 under UV-B have been suggested. In addition, progress has been made in understanding UVR8-regulated gene expression, and an increasing number of physiological responses are being linked to UVR8 signaling, including cross-regulation with responses induced by distinct environmental signals. However, many unknown aspects of UVR8 signaling await further investigation, including how UVR8 regulates gene transcription in the nucleus, the nature of COP1 signaling in the UVR8 pathway, and details of the link between UVR8 activation and repression of hormonal biosynthesis and/or signaling. Further study in these areas should increase our understanding of the UVR8 UV-B signaling pathway and the interplay between UV-B and other environmental factors.

\section{Acknowledgments}

We apologize to our colleagues whose work we have not included in this manuscript due to space restrictions. Our research is supported by the University of Geneva, the Swiss National Science Foundation (grants no. 31003A_153475 and CRSII3_154438) and the European Research Council (ERC) Grant 310539 (UV-B Perception) under the European Union's Seventh Framework Programme. 


\section{References and recommended reading}

Papers of particular interest, published within the period of review (2015 \& 2016), have been highlighted as:

- of special interest

$\bullet$ of outstanding interest

1. Rizzini L, Favory JJ, Cloix C, Faggionato D, O'Hara A, Kaiserli E, Baumeister R, Schafer E, Nagy F, Jenkins GI, et al.: Perception of UV-B by the Arabidopsis UVR8 protein. Science 2011, 332:103-106.

2. Galvao VC, Fankhauser C: Sensing the light environment in plants: photoreceptors and early signaling steps. Curr Opin Neurobiol 2015, 34:46-53.

3. González Besteiro MA, Bartels S, Albert A, Ulm R: Arabidopsis MAP kinase phosphatase 1 and its target MAP kinases 3 and 6 antagonistically determine UVB stress tolerance, independent of the UVR8 photoreceptor pathway. Plant J 2011, 68:727-737.

4. Tilbrook K, Arongaus AB, Binkert M, Heijde M, Yin R, Ulm R: The UVR8 UV-B photoreceptor: perception, signaling and response. Arabidopsis Book 2013, 11:e0164.

5. Jenkins GI: Structure and function of the UV-B photoreceptor UVR8. Curr Opin Struct Biol 2014, 29:52-57.

6. Ulm R, Jenkins GI: Q\&A: How do plants sense and respond to UV-B radiation? $B M C$ Biol 2015, 13:45.

7. Yang X, Montano S, Ren Z: How does photoreceptor UVR8 perceive a UV-B signal? Photochem Photobiol 2015, 91:993-1003.

8. Kliebenstein DJ, Lim JE, Landry LG, Last RL: Arabidopsis UVR8 regulates ultravioletB signal transduction and tolerance and contains sequence similarity to human regulator of chromatin condensation 1. Plant Physiol 2002, 130:234-243.

9. Brown BA, Cloix C, Jiang GH, Kaiserli E, Herzyk P, Kliebenstein DJ, Jenkins GI: A UVB-specific signaling component orchestrates plant UV protection. Proc Natl Acad Sci USA 2005, 102:18225-18230.

10. Favory JJ, Stec A, Gruber H, Rizzini L, Oravecz A, Funk M, Albert A, Cloix C, Jenkins GI, Oakeley EJ, et al.: Interaction of COP1 and UVR8 regulates UV-B-induced photomorphogenesis and stress acclimation in Arabidopsis. EMBO $J$ 2009, 28:591-601. 
11. Morales LO, Brosche M, Vainonen J, Jenkins GI, Wargent JJ, Sipari N, Strid A, Lindfors AV, Tegelberg R, Aphalo PJ: Multiple roles for UV RESISTANCE LOCUS8 in regulating gene expression and metabolite accumulation in Arabidopsis under solar ultraviolet radiation. Plant Physiol 2013, 161:744-759.

12. Huang X, Yang P, Ouyang X, Chen L, Deng XW: Photoactivated UVR8-COP1 module determines photomorphogenic UV-B signaling output in Arabidopsis. PLoS Genet 2014, 10:e1004218.

13. Stracke R, Favory JJ, Gruber H, Bartelniewoehner L, Bartels S, Binkert M, Funk M, Weisshaar B, Ulm R: The Arabidopsis bZIP transcription factor HY5 regulates expression of the PFG1/MYB12 gene in response to light and ultraviolet-B radiation. Plant Cell Environ 2010, 33:88-103.

14. Davey MP, Susanti NI, Wargent JJ, Findlay JE, Paul Quick W, Paul ND, Jenkins GI: The UV-B photoreceptor UVR8 promotes photosynthetic efficiency in Arabidopsis thaliana exposed to elevated levels of UV-B. Photosynth Res 2012, 114:121-131.

15. Li N, Teranishi M, Yamaguchi H, Matsushita T, Watahiki MK, Tsuge T, Li SS, Hidema J: UV-B-induced CPD photolyase gene expression is regulated by UVR8-dependent and -independent pathways in Arabidopsis. Plant Cell Physiol 2015, 56:2014-2023.

16. Feher B, Kozma-Bognar L, Kevei E, Hajdu A, Binkert M, Davis SJ, Schafer E, Ulm R, Nagy F: Functional interaction of the circadian clock and UV RESISTANCE LOCUS 8-controlled UV-B signaling pathways in Arabidopsis thaliana. Plant J 2011, 67:37-48.

17. Vandenbussche F, Tilbrook K, Fierro AC, Marchal K, Poelman D, Van Der Straeten D, Ulm R: Photoreceptor-mediated bending towards UV-B in Arabidopsis. Mol Plant 2014, 7:1041-1052.

18. Fierro AC, Leroux O, De Coninck B, Cammue BP, Marchal K, Prinsen E, Van Der Straeten D, Vandenbussche F: Ultraviolet-B radiation stimulates downward leaf curling in Arabidopsis thaliana. Plant Physiol Biochem 2015, 93:9-17.

19. Tossi V, Lamattina L, Jenkins GI, Cassia RO: Ultraviolet-B-induced stomatal closure in Arabidopsis is regulated by the UV RESISTANCE LOCUS8 photoreceptor in a nitric oxide-dependent mechanism. Plant Physiol 2014, 164:2220-2230.

20. Demkura PV, Ballare CL: UVR8 mediates UV-B-induced Arabidopsis defense responses against Botrytis cinerea by controlling sinapate accumulation. Mol Plant 2012, 5:116-126. 
21. Wargent JJ, Gegas VC, Jenkins GI, Doonan JH, Paul ND: UVR8 in Arabidopsis thaliana regulates multiple aspects of cellular differentiation during leaf development in response to ultraviolet B radiation. New Phytol 2009, 183:315-326.

22. Fasano R, Gonzalez N, Tosco A, Dal Piaz F, Docimo T, Serrano R, Grillo S, Leone A, Inze D: Role of Arabidopsis UV RESISTANCE LOCUS 8 in plant growth reduction under osmotic stress and low levels of UV-B. Mol Plant 2014, 7:773-791. 23••. Hayes S, Sharma A, Fraser DP, Trevisan M, Cragg-Barber CK, Tavridou E, Fankhauser C, Jenkins GI, Franklin KA: UV-B perceived by the UVR8 photoreceptor inhibits plant thermomorphogenesis. Curr Biol 2017, 27:120-127.

This study presents a novel role of UVR8 in antagonizing high temperature-induced stem elongation via inhibition of PIF4 activity.

24. Hayes S, Velanis CN, Jenkins GI, Franklin KA: UV-B detected by the UVR8 photoreceptor antagonizes auxin signaling and plant shade avoidance. Proc Natl Acad Sci USA 2014, 111:11894-11899.

25. Mazza CA, Ballare CL: Photoreceptors UVR8 and phytochrome B cooperate to optimize plant growth and defense in patchy canopies. New Phytol 2015, 207:4-9.

26••. Allorent G, Lefebvre-Legendre L, Chappuis R, Kuntz M, Truong TB, Niyogi KK, Ulm R, Goldschmidt-Clermont M: UV-B photoreceptor-mediated protection of the photosynthetic machinery in Chlamydomonas reinhardtii. Proc Natl Acad Sci USA 2016, 113:14864-14869.

This work discovered a distinct regulatory link between Chlamydomonas UVR8 and nonphotochemical quenching-based photoprotection in the chloroplast. This pathway is complementary to the phototropin-based high light response described in [28].

27•. Tilbrook K, Dubois M, Crocco CD, Yin R, Chappuis R, Allorent G, Schmid-Siegert E, Goldschmidt-Clermont $\mathrm{M}$, Ulm R: UV-B perception and acclimation in Chlamydomonas reinhardtii. Plant Cell 2016, 28:966-983.

This work describes the evolutionary conservation of the UVR8-COP1-mediated signaling pathway in the unicellular alga Chlamydomonas reinhardtii and of the importance of this pathway for UV-B acclimation.

28. Petroutsos D, Tokutsu R, Maruyama S, Flori S, Greiner A, Magneschi L, Cusant L, Kottke T, Mittag M, Hegemann P, et al.: A blue-light photoreceptor mediates the feedback regulation of photosynthesis. Nature 2016, 537:563-566.

29. Puchta H: Applying CRISPR/Cas for genome engineering in plants: the best is yet to come. Curr Opin Plant Biol 2017, 36:1-8. 
30. Christie JM, Arvai AS, Baxter KJ, Heilmann M, Pratt AJ, O'Hara A, Kelly SM, Hothorn M, Smith BO, Hitomi K, et al.: Plant UVR8 photoreceptor senses UV-B by tryptophan-mediated disruption of cross-dimer salt bridges. Science 2012, 335:1492-1496.

31. Wu D, Hu Q, Yan Z, Chen W, Yan C, Huang X, Zhang J, Yang P, Deng H, Wang J, et al.: Structural basis of ultraviolet-B perception by UVR8. Nature 2012, 484:214-219.

32. Zeng X, Ren Z, Wu Q, Fan J, Peng P-P, Tang K, Zhang R, Zhao K-H, Yang X: Dynamic crystallography reveals early signalling events in ultraviolet photoreceptor UVR8. Nature Plants 2015, 1:14006.

33. Liu Z, Li X, Zhong FW, Li J, Wang L, Shi Y, Zhong D: Quenching dynamics of ultraviolet-light perception by UVR8 photoreceptor. J Phys Chem Lett 2014, 5:6972.

34. Voityuk AA, Marcus RA, Michel-Beyerle ME: On the mechanism of photoinduced dimer dissociation in the plant UVR8 photoreceptor. Proc Natl Acad Sci USA 2014, 111:5219-5224.

35. Wu M, Strid A, Eriksson LA: Photochemical reaction mechanism of UV-B-induced monomerization of UVR8 dimers as the first signaling event in UV-B-regulated gene expression in plants. $J$ Phys Chem $B$ 2014, 118:951-965.

36. Mathes T, Heilmann M, Pandit A, Zhu J, Ravensbergen J, Kloz M, Fu Y, Smith BO, Christie JM, Jenkins GI, et al.: Proton-coupled electron transfer constitutes the photoactivation mechanism of the plant photoreceptor UVR8. J Am Chem Soc 2015, 137:8113-8120.

37. Heilmann M, Velanis CN, Cloix C, Smith BO, Christie JM, Jenkins GI: Dimer/monomer status and in vivo function of salt-bridge mutants of the plant UV-B photoreceptor UVR8. Plant J 2016, 88:71-81.

38. Heilmann M, Christie JM, Kennis JT, Jenkins GI, Mathes T: Photoinduced transformation of UVR8 monitored by vibrational and fluorescence spectroscopy. Photochem Photobiol Sci 2015, 14:252-257.

39. Miyamori T, Nakasone Y, Hitomi K, Christie JM, Getzoff ED, Terazima M: Reaction dynamics of the UV-B photosensor UVR8. Photochem Photobiol Sci 2015, 14:9951004.

40. Findlay KM, Jenkins GI: Regulation of UVR8 photoreceptor dimer/monomer photoequilibrium in Arabidopsis plants grown under photoperiodic conditions. Plant Cell Environ 2016, 39:1706-1714. 
41. Yin R, Arongaus AB, Binkert M, Ulm R: Two distinct domains of the UVR8 photoreceptor interact with COP1 to initiate UV-B signaling in Arabidopsis. Plant Cell 2015, 27:202-213.

This work shows that UVR8 interacts with the COP1 WD40 domain via the $\beta$-propeller core domain and the C-terminal C27 domain, proposing a two-step model for UVR8-mediated COP1 interaction and COP1 regulation.

42. O'Hara A, Jenkins GI: In vivo function of tryptophans in the Arabidopsis UV-B photoreceptor UVR8. Plant Cell 2012, 24:3755-3766.

43. Cloix C, Kaiserli E, Heilmann M, Baxter KJ, Brown BA, O'Hara A, Smith BO, Christie JM, Jenkins GI: C-terminal region of the UV-B photoreceptor UVR8 initiates signaling through interaction with the COP1 protein. Proc Natl Acad Sci USA 2012, 109:16366-16370.

44. Heijde M, Binkert M, Yin R, Ares-Orpel F, Rizzini L, Van De Slijke E, Persiau G, Nolf J, Gevaert K, De Jaeger G, et al.: Constitutively active UVR8 photoreceptor variant in Arabidopsis. Proc Natl Acad Sci USA 2013, 110:20326-20331.

45. Lau OS, Deng XW: The photomorphogenic repressors COP1 and DET1: 20 years later. Trends Plant Sci 2012, 17:584-593.

46. Oravecz A, Baumann A, Mate Z, Brzezinska A, Molinier J, Oakeley EJ, Adam E, Schafer E, Nagy F, Ulm R: CONSTITUTIVELY PHOTOMORPHOGENIC1 is required for the UV-B response in Arabidopsis. Plant Cell 2006, 18:1975-1990.

47. Brown BA, Jenkins GI: UV-B signaling pathways with different fluence-rate response profiles are distinguished in mature Arabidopsis leaf tissue by requirement for UVR8, HY5, and HYH. Plant Physiol 2008, 146:576-588.

48. Binkert M, Kozma-Bognar L, Terecskei K, De Veylder L, Nagy F, Ulm R: UV-Bresponsive association of the Arabidopsis bZIP transcription factor ELONGATED HYPOCOTYL5 with target genes, including its own promoter. Plant Cell 2014, 26:4200-4213.

49. Ulm R, Baumann A, Oravecz A, Mate Z, Adam E, Oakeley EJ, Schafer E, Nagy F: Genome-wide analysis of gene expression reveals function of the bZIP transcription factor HY5 in the UV-B response of Arabidopsis. Proc Natl Acad Sci USA 2004, 101:1397-1402.

50. Gruber H, Heijde M, Heller W, Albert A, Seidlitz HK, Ulm R: Negative feedback regulation of UV-B-induced photomorphogenesis and stress acclimation in Arabidopsis. Proc Natl Acad Sci USA 2010, 107:20132-20137. 
51. Heijde M, Ulm R: Reversion of the Arabidopsis UV-B photoreceptor UVR8 to the homodimeric ground state. Proc Natl Acad Sci USA 2013, 110:1113-1118.

52. Uljon S, Xu X, Durzynska I, Stein S, Adelmant G, Marto JA, Pear WS, Blacklow SC: Structural basis for substrate selectivity of the E3 ligase COP1. Structure 2016, 24:687-696.

53. Wu M, Eriksson LA, Strid A: Theoretical prediction of the protein-protein interaction between Arabidopsis thaliana COP1 and UVR8. Theor Chem Acc 2013, 132:1371.

54. Holm M, Hardtke CS, Gaudet R, Deng XW: Identification of a structural motif that confers specific interaction with the WD40 repeat domain of Arabidopsis COP1. EMBO J 2001, 20:118-127.

55. Huang X, Ouyang X, Yang P, Lau OS, Chen L, Wei N, Deng XW: Conversion from CUL4-based COP1-SPA E3 apparatus to UVR8-COP1-SPA complexes underlies a distinct biochemical function of COP1 under UV-B. Proc Natl Acad Sci USA 2013, 110:16669-16674.

56. Kaiserli E, Jenkins GI: UV-B promotes rapid nuclear translocation of the Arabidopsis UV-B specific signaling component UVR8 and activates its function in the nucleus. Plant Cell 2007, 19:2662-2673.

57•. Yin R, Skvortsova MY, Loubery S, Ulm R: COP1 is required for UV-B-induced nuclear accumulation of the UVR8 photoreceptor. Proc Natl Acad Sci USA 2016, 113:E4415-4422.

Together with [58•] this work shows that nuclear UVR8 is required for UV-B signaling, and discovered a role of COP1 in regulating UV-B induced accumulation of UVR8.

58•. Qian C, Mao W, Liu Y, Ren H, Lau OS, Ouyang X, Huang X: Dual-source nuclear monomers of UV-B light receptor direct photomorphogenesis in Arabidopsis. $\mathrm{Mol}$ Plant 2016, 9:1671-1674.

Together with [57•] this work shows that nuclear UVR8 is required for UV-B signaling, and discovered a role of COP1 in regulating UV-B induced accumulation of UVR8.

59. Stacey MG, Hicks SN, von Arnim AG: Discrete domains mediate the light-responsive nuclear and cytoplasmic localization of Arabidopsis COP1. Plant Cell 1999, 11:349-364.

60. Velanis CN, Herzyk P, Jenkins GI: Regulation of transcription by the Arabidopsis UVR8 photoreceptor involves a specific histone modification. Plant Mol Biol 2016, 92:425-443. 
61. Cloix C, Jenkins GI: Interaction of the Arabidopsis UV-B-specific signaling component UVR8 with chromatin. Mol Plant 2008, 1:118-128.

62. Binkert M, Crocco CD, Ekundayo B, Lau K, Raffelberg S, Tilbrook K, Yin R, Chappuis R, Schalch T, Ulm R: Revisiting chromatin binding of the Arabidopsis UV-B photoreceptor UVR8. BMC Plant Biol 2016, 16:42.

63. Huang X, Ouyang X, Yang P, Lau OS, Li G, Li J, Chen H, Deng XW: Arabidopsis FHY3 and HY5 positively mediate induction of COP1 transcription in response to photomorphogenic UV-B light. Plant Cell 2012, 24:4590-4606.

64. Holm M, Ma LG, Qu LJ, Deng XW: Two interacting bZIP proteins are direct targets of COP1-mediated control of light-dependent gene expression in Arabidopsis. Genes Dev 2002, 16:1247-1259.

65. Jiang L, Wang Y, Li QF, Bjorn LO, He JX, Li SS: Arabidopsis STO/BBX24 negatively regulates UV-B signaling by interacting with COP1 and repressing HY5 transcriptional activity. Cell Res 2012, 22:1046-1057.

66. Hornitschek P, Lorrain S, Zoete V, Michielin O, Fankhauser C: Inhibition of the shade avoidance response by formation of non-DNA binding bHLH heterodimers. EMBO J 2009, 28:3893-3902.

67. Fernandez MB, Tossi V, Lamattina L, Cassia R: A comprehensive phylogeny reveals functional conservation of the UV-B photoreceptor UVR8 from green algae to higher plants. Front Plant Sci 2016, 7:1698.

68. Olsen JL, Rouze P, Verhelst B, Lin YC, Bayer T, Collen J, Dattolo E, De Paoli E, Dittami S, Maumus F, et al.: The genome of the seagrass Zostera marina reveals angiosperm adaptation to the sea. Nature 2016, 530:331-335. 
491 Figure 1. Selected physiological roles of UVR8-mediated UV-B signaling. Further details 492 and references can be found in the text.

493

494 Figure 2. Working models for UV-B-triggered and COP1-dependent nuclear accumulation of 495 UVR8.

496 UVR8 homodimers perceive UV-B through their intrinsic tryptophan chromophores and then 497 dissociate to their active monomer conformation. Import model: UVR8 monomers bind to 498 COP1 in the cytosol and the UVR8-COP1 heterocomplexes enter the nucleus by COP1 NLS499 mediated co-import ("piggy back"). Retention model: UVR8 monomers enter the nucleus 500 through interaction and co-import with a presently unknown protein providing an NLS signal, 501 or by diffusion independent of a helper protein. However, nuclear COP1 interaction with 502 UVR8 is required to prevent nuclear exit of UVR8. Nuclear UVR8-COP1 interaction leads to 503 HY5 stabilization and enhanced binding of HY5 to promoter regions of UV-B-responsive 504 genes. RUP1 and RUP2 facilitate the reversion of UVR8 monomers to homodimers, likely in 505 the nucleus as well as cytosol (for simplicity only cytosol shown).

506

507 


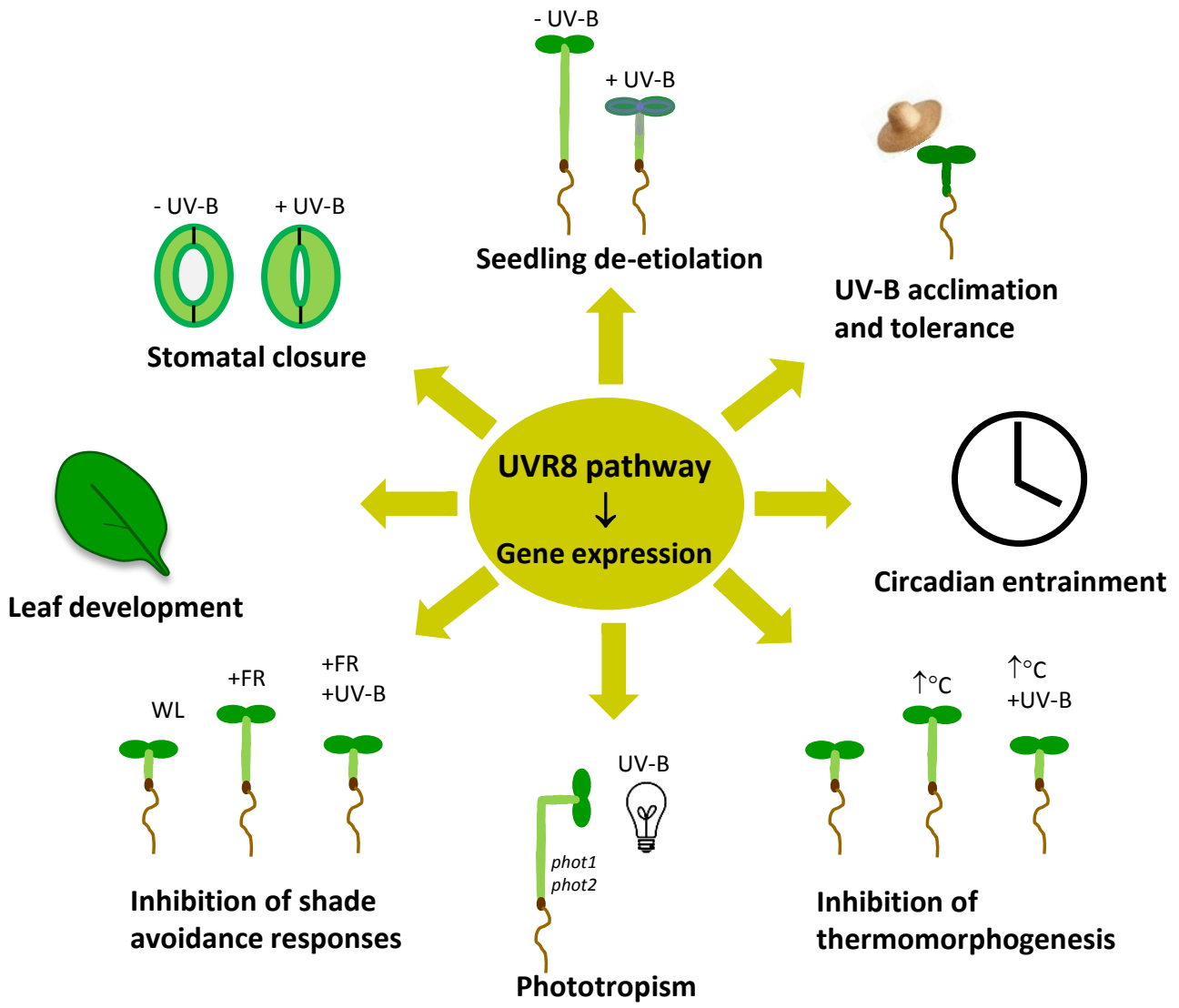



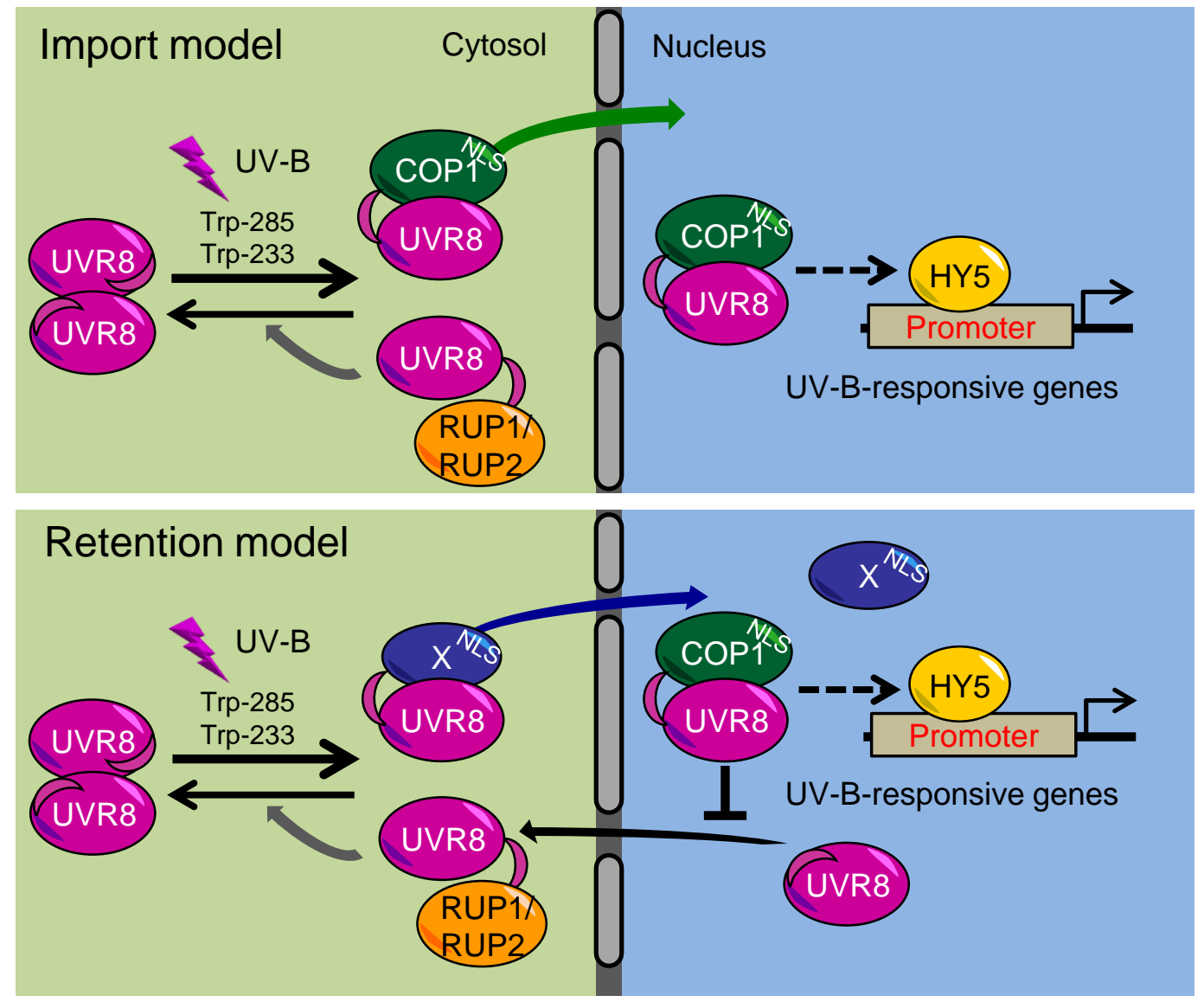